\title{
A Rough Set Based Decision Tree Algorithm and Its Application in Intrusion Detection
}

\author{
Lin Zhou and Feng Jiang \\ College of Information Science and Technology, Qingdao University of Science and \\ Technology, Qingdao 266061, P.R. China
}

\begin{abstract}
In this paper, we propose a novel rough set based algorithm to induce decision trees. To improve the computation efficiency of our algorithm, the rough set based attribute reduction technology is used to filter out the irrelevant attributes from the original set of attributes. And the notions of the significance of attribute and the uniformity of attribute in rough sets are adopted as the heuristic information for the selection of splitting attributes. Moreover, we apply the proposed algorithm to intrusion detection. The experimental results demonstrate that our algorithm can provide competitive solutions efficiently.
\end{abstract}

Keywords: Rough sets, decision trees, significance of attribute, uniformity of attribute, intrusion detection.

\section{Introduction}

Decision tree algorithms are used extensively in data mining [1-2]. Since most of the current algorithms use all attributes to construct decision trees, and do not concern the relevancy among attributes [1-2]. This may lead to extra overhead in terms of memory and computational efforts [5-6]. To solve that problem, rough set-based decision tree algorithms have been proposed within last few years [3-6].

Intrusion detection systems (IDS) have become an essential component of computer security. The concept of IDS was first introduced by Anderson in 1980 [7]. Due to the large volumes of security audit data as well as complex and dynamic properties of intrusion behaviors, to optimize the performance of IDS, many artificial intelligence techniques have been utilized, where decision tree technology plays an important role in IDS [8].

In this paper, we propose a novel rough set based decision tree algorithm RSDT and apply it to IDS. In our algorithm, the rough set based attribute reduction technology is used to filter out redundant information, which is crucial to IDS. Moreover, we introduce a new concept called the uniformity for each attribute in a given decision table. And we use the significance of attribute and the uniformity of attribute as the heuristic information for the selection of splitting attributes. Numerical experiments show that our algorithm is efficient for intrusion detection. 


\section{Preliminaries}

In rough sets, a decision table is a 5-ruple $D T=(U, C, D, V, f)$, where $U$ is the set of objects; $C$ is the set of condition attributes; $D$ is the set of decision attributes; $V=\bigcup_{a \in C \cup D} V_{a}$ is the union of attribute domains; $f: U \times(C \cup D) \rightarrow V$ is a function such that for any $a \in C \cup D$ and $x \in U, f(x, a) \in V_{a}[4,9]$.

Given a decision table $D T=(U, C, D, V, f)$, let $B \subseteq C \cup D$, we call equivalence relation $I N D(B)$ an indiscernibility relation, which is defined as $\operatorname{IND}(B)=$ $\{(x, y) \in U \times U: \forall a \in B(f(x, a)=f(y, a))\} . I N D(B)$ partitions $U$ into disjoint equivalence classes, let $U / I N D(B)$ denote the family of all equivalence classes of $I N D(B)$. For simplicity, $U / B$ will be written instead of $U / I N D(B)[4,9]$.

Definition 1 [Positive Region]. Given a decision table $D T=(U, C, D, V, f)$, for any $B \subseteq C$, the positive region of $D$ with respect to $B$ is defined as $[4,9]$ :

$$
\operatorname{POS}_{B}(D)=\bigcup_{E \in U / B \wedge \forall x, y \in E((x, y) \in I N D(D))} E,
$$

where $E$ is an equivalence class in the partition $U / B$.

Definition 2 [Significance of Attribute]. Given a decision table $D T=(U, C$, $D, V, f)$, let $B \subseteq C$, for any $a \in B$, the significance of attribute a with respect to $B$ and $D$ is defined as follows $[4,9]$ :

$$
S G F(a, B, D)=P O S_{B}(D)-P O S_{B-\{a\}}(D) .
$$

\section{RSDT Algorithm and Its Application in IDS}

Definition 3 [Uniformity of Attribute] Given a decision table $D T=(U, C$, $D, V, f)$, for any $a \in C \cup D$, let $U /\{a\}=\left\{E_{1}, \ldots, E_{k}\right\}$ denote the partition induced by relation $I N D(\{a\})$. The uniformity of attribute a is defined as:

$$
U N(a)=\frac{\left(\left|E_{1}\right|-A v e\right)^{2}+\ldots+\left(\left|E_{k}\right|-A v e\right)^{2}}{k},
$$

where Ave $=\frac{\left|E_{1}\right|+\ldots+\left|E_{k}\right|}{k}$.

Algorithm 1. RSDT.

Input: decision table $T_{1}=(U, C, D, V, f)$, i.e., the training set. Output: set $R$ of decision rules.

\section{Function Main $\left(T_{1}\right)$}

(1) For each continuous attribute $c \in C$, discretize $c$ by virtue of a discretization approach [9]. Let $T_{2}=\left(U_{2}, C, D, V_{2}, f_{2}\right)$ denote the discretized dataset. 
(2) Compute the reduct Red of $C$ with respect to $D$ in $T_{2}$, using a rough set based attribute reduction approach $[9-11,16]$.

(3) Reduce $T_{2}$ based on the reduct Red. Let $T_{3}=\left(U_{3}, R e d, D, V_{3}, f_{3}\right)$ denote the reduced dataset.

(4) Decision_Tree $\left(T_{3}\right)$. //Call a function defined below to induce a decision tree.

(5) Generate a set $R$ of rules by traversing all the paths from the root to the leaf node in the decision tree.

(6) Return $R$.

Function Decision_Tree $\left(T_{\text {current }}\right) \quad / / T_{\text {current }}=\left(U^{\prime}, C^{\prime}, D, V^{\prime}, f^{\prime}\right)$ is the decision table that is currently in use.

(1) For each attribute $a \in C^{\prime}$, calculate the significance $\operatorname{SGF}\left(a, C^{\prime}, D\right)$ of a with respect to $C^{\prime}$ and $D$ in $T_{\text {current }}$.

(2) Select $t$ from $C^{\prime}$ with the largest significance as the splitting attribute.

(3) If there exist more than one attributes with the largest significance, then calculate the uniformity for each of these attributes in $T_{\text {current }}$, and select attribute $t$ with the largest uniformity (If there still exist more than one attributes with the largest uniformity, then arbitrarily select one from them).

(4) Create a node $N$, label $N$ with attribute $t$, and let $C^{\prime}=C^{\prime}-\{t\}$.

(5) For each value $v_{j}$ of attribute $t$

(5.1) Generate one branch of $N$, labeled with $t=v_{j}$;

(5.2) Let $S_{j}=\left\{o \in U^{\prime}: f^{\prime}(o, t)=v_{j}\right\}$;

(5.3) If $S_{j}=\emptyset$, then add one leaf node $L$ labeled with the most common class in $U^{\prime}$;

(5.4) If $S_{j} \neq \emptyset$, and all objects in $S_{j}$ belong to the same class $d$, then add one leaf node $L$ labeled with the class $d$;

(5.5) If $S_{j} \neq \emptyset, C^{\prime}=\emptyset$, and objects in $S_{j}$ belong to different classes, then add a leaf node $L$ labeled with the most common class in $S_{j}$.

(5.6) If $S_{j} \neq \emptyset, C^{\prime} \neq \emptyset$, and objects in $S_{j}$ belong to different classes, then obtain a sub-table $T_{\text {sub }}=\left\{S_{j}, C^{\prime}, D, V_{\text {sub }}, f_{\text {sub }}\right\}$ of $T_{\text {current }}$, and call function Decision_Tree $\left(T_{\text {sub }}\right)$ to construct a sub-tree.

In algorithm 1 , before computing the partition $U^{\prime} / C^{\prime}$, we first sort all objects of $U^{\prime}$ based on the counting sort [11], then we can calculate $U^{\prime} / C^{\prime}$ in $O(m \times$ $n$ ) time, where $m=\left|C^{\prime}\right|, n=\left|U^{\prime}\right|$. Hence, we can calculate the significance $S G F\left(a, C^{\prime}, D\right)$ of $a$ in $O(m \times n)$ time. Therefore, in the worst case, the time complexity of step (4) in function Main is $O\left(|R e d|^{3} \times\left|U_{3}\right|\right)$. And the overall time complexity of algorithm 1 is also determined by that of the discretization approach and that of the attribute reduction approach in Main [9-11, 16].

\section{Experiment}

To evaluate the performance of RSDT algorithm, we ran it on KDD-99 dataset [12]. Experiments were conducted on a $2.0 \mathrm{GHz}$ Pentium 4 machine with $2 \mathrm{~GB}$ RAM. We compared algorithm RSDT with algorithms ID3 and C4.5 [1-2]. RSDT was implemented in Pascal. And ID3 and C4.5 are available in Weka [13]. 
Since the full KDD-99 training dataset is too large for our purposes, a concise subset of KDD-99, known as '10\%KDD', will be discussed here. The $10 \%$ KDD dataset contains 22 different attack types and normal records. The 22 attack types fall into four main categories [12]: PROBE, DoS, U2R and R2L.

Although the $10 \% \mathrm{KDD}$ dataset is a concise subset of the full KDD-99 training dataset, it contains 494021 records [12], which is still very large for our purposes. Here we follow the experimental technique of Chen et al. [14], which does random sampling without replacement from the $10 \% \mathrm{KDD}$ dataset to form a smaller dataset called 'Final_Dataset'. The following table gives the numbers of instances that are selected for different attack types and normal records.

Table 1. The numbers of instances selected

\begin{tabular}{lll||lll}
\hline $\begin{array}{l}\text { Attack } \\
\text { Type }\end{array}$ & $\begin{array}{l}\text { Number of } \\
\text { Instances }\end{array}$ & $\begin{array}{l}\text { Number of Ins- } \\
\text { tances Selected }\end{array}$ & $\begin{array}{l}\text { Attack } \\
\text { Type }\end{array}$ & $\begin{array}{l}\text { Number of } \\
\text { Instances }\end{array}$ & $\begin{array}{l}\text { Number of Ins- } \\
\text { tances Selected }\end{array}$ \\
\hline back & 2203 & 220 & perl & 3 & 3 \\
\hline buffer_overflow & 30 & 30 & phf & 4 & 4 \\
\hline ftp_write & 8 & 8 & pod & 264 & 264 \\
\hline guess_passwd & 53 & 53 & portsweep & 1040 & 104 \\
\hline imap & 12 & 12 & rootkit & 10 & 10 \\
\hline ipsweep & 1247 & 125 & satan & 1589 & 160 \\
\hline land & 21 & 21 & smurf & 280790 & 2808 \\
\hline loadmodule & 9 & 9 & spy & 2 & 2 \\
\hline multihop & 7 & 7 & teardrop & 979 & 100 \\
\hline neptune & 107201 & 1072 & warezclient & 1020 & 102 \\
\hline nmap & 231 & 231 & warezmaster & 20 & 20 \\
\hline normal & 97278 & 9730 & Total & 494021 & 15095 \\
\hline
\end{tabular}

There are four steps in our experiments:

(I) The first step is to prepare the dataset. As mentioned above, we do random sampling without replacement from the $10 \% \mathrm{KDD}$ dataset to obtain a new dataset called Final_Dataset.

(II) Next is the discretization step. We use two simple discretization algorithms: Equal Width Binning (EW) and Equal Frequency Binning (EF), to respectively discretize the continuous attributes in Final_Dataset [9]. EW and EF algorithms are also available in Weka [13]. And for both of the two algorithms, the numbers of bins are set to 3 . It should be noted that although Final_Dataset contains 34 continuous attributes, we only discretize the following 26 attributes of them: (1) Duration; (2) Src_bytes; (3) Dst_bytes; (4) Hot; (5) Num_compromised; (6) Num_root; (7) Num_file_creations; (8) Count; (9) Srv_count; (10) Serror_rate; (11) Srv_serror_rate; (12) Rerror_rate; (13) Srv_rerror _rate; (14) Same_srv_rate; (15) Diff_srv_rate; (16) Srv_diff_host_rate; (17) Dst_host _count; (18) Dst_host_ srv_count; (19) Dst_host_ same_srv_rate; (20) Dst_host_diff_ srv_rate; (21) Dst_ host_same_src_port_ rate; (22) Dst_host_srv_diff_ host_rate; (23) Dst_host_ serror_rate; (24) Dst_host_srv_serror_rate; (25) Dst_host_rerror_rate; (26) Dst_ host_srv_ rerror_rate. 
Moreover, each discretized Final_Dataset is randomly divided into a training dataset $(60 \%$ of the data) and a test dataset ( $40 \%$ of the data).

(III) The third step is constructing decision trees on training datasets using algorithms RSDT, ID3 and C4.5, respectively. Then different sets of rules can be generated from these trees. It should be noted that before applying RSDT to each training dataset, we first reduce the dataset by virtue of the attribute reduction algorithm proposed by Liu et al. [10].

(IV) The last step is applying the rules generated in (III) to classify the test dataset based on a given classifier. For ID3 and C4.5, the process is also implemented in Weka [13]. And for RSDT, we adopt the Standard Voting classifier available in Rosetta [15]. The corresponding options are: CLASSIFIER = StandardVoter; FALLBACK= True; FALLBACK.CLASS=DoS.

Tables 2 details the classification results of different decision tree algorithms on the dataset discretized by EW algorithm.

Table 2. The classification results on the dataset discretized by EW

\begin{tabular}{llll}
\hline $\begin{array}{l}\text { Decision Tree } \\
\text { Algorithm }\end{array}$ & $\begin{array}{l}\text { Number of } \\
\text { Attributes }\end{array}$ & $\begin{array}{l}\text { Classification } \\
\text { Accuracy(\%) }\end{array}$ & $\begin{array}{l}\text { Time Taken to } \\
\text { Build Model(s) }\end{array}$ \\
\hline RSDT & 24 & 97.88 & 0.297 \\
\hline ID3 & 41 & 97.665 & 1.06 \\
\hline C4.5 & 41 & 97.582 & 1.63 \\
\hline
\end{tabular}

From Table 2, we can see that for the dataset discretized by EW, RSDT algorithm perform better than ID3 and C4.5. Our algorithm generates a better rule learning scheme for IDS than the other two algorithms, since the detection rate (i.e., classification accuracy) of our algorithm is obviously higher than those of other algorithms, while the training time (i.e., time taken to build model) of our algorithm is much lower than those of other algorithms.

Moreover, the following table details the classification results of different decision tree algorithms on the dataset discretized by EF algorithm.

Table 3. The classification results on the dataset discretized by EF

\begin{tabular}{llll}
\hline $\begin{array}{l}\text { Decision Tree } \\
\text { Algorithm }\end{array}$ & $\begin{array}{l}\text { Number of } \\
\text { Attributes Used }\end{array}$ & $\begin{array}{l}\text { Classification } \\
\text { Accuracy(\%) }\end{array}$ & $\begin{array}{l}\text { Time Taken to } \\
\text { Build Model(s) }\end{array}$ \\
\hline RSDT & 15 & 99.255 & 0.079 \\
\hline ID3 & 41 & 99.023 & 0.83 \\
\hline C4.5 & 41 & 98.924 & 0.74 \\
\hline
\end{tabular}

From Table 3, it is easy to see that for the dataset discretized by EF, RSDT algorithm also has the best performance. Hence, this experiment also demonstrates the effectiveness of our decision tree algorithm for intrusion detection.

\section{Conclusion}

In this paper, a novel algorithm based on the significance of attribute and the uniformity of attribute in rough set theory was proposed for constructing decision 
trees. Differing from traditional algorithms, our algorithm employed a new kind of heuristic information for the selection of splitting attributes. And the positive region based attribute reduction technology was used to execute pre-pruning for decision trees, thus the attributes which are not correlated with the decision information are deleted and the total nodes of decision trees are limited. Hence, our algorithm is suitable to deal with the mass data in IDS. Experimental results on the KDD-99 dataset showed that our algorithm generated a better rule learning scheme for IDS than other algorithms.

\section{Acknowledgements}

This work is supported by the National Natural Science Foundation of China (grant nos. 60802042, 61035004), and the Natural Science Foundation of Shandong Province, China (grant nos. ZR2009GQ013, ZR2010FQ027).

\section{References}

1. Quinlan, R.: Induction of decision trees. Machine Learning 1(1), 81-106 (1986)

2. Quinlan, R.: C4.5: Programs for Machine Learning. Morgan Kaufmann, San Francisco (1993)

3. Pawlak, Z.: Rough Sets. Int. J. Comput. Informat. Sci. 11(5), 341-356 (1982)

4. Pawlak, Z.: Rough Sets: Theoretical Aspects of Reasoning about Data. Kluwer Academic Publishing, Dordrecht (1991)

5. Li, X.P., Dong, M.: An algorithm for constructing decision tree based on variable precision rough set model. In: Proc. of the 4th Int. Conf. on Natural Computation, pp. 280-283 (2008)

6. Jiang, Y., Li, Z.H., Zhang, Q., Liu, Y.: New method for constructing decision tree based on rough set theory. Computer Appliactions 24(8), 21-23 (2004)

7. Anderson, J.P.: Computer Security Threat Monitoring and Surveillance. James P. Anderson Co., Fort Washington (1980)

8. Li, X.Y., Ye, N.: Decision tree classifiers for computer intrusion detection. Journal of Parallel and Distributed Computing Practices 4(2), 179-190 (2001)

9. Wang, G.Y.: Rough set theory and knowledge acquisition. Xian Jiaotong University Press (2001)

10. Liu, S.H., Sheng, Q.J., Wu, B., Shi, Z.Z.: Research on efficient algorithms for rough set methods. Chinese Journal of Computers 26(5), 525-529 (2003)

11. Xu, Z.Y., Liu, Z.P., Yang, B.R., Song, W.: A Quick Attribute Reduction Algorithm with Complexity of $\max \left(O(|C||U|), O\left(|C|^{2}|U / C|\right)\right)$. Chinese Journal of Computers 29(3), 391-399 (2006)

12. KDD Cup 99 Dataset (1999), http://kdd.ics.uci.edu/databases/kddcup99/kddcup99.html

13. Witten, I.H., Frank, E.: Data Mining: Practical Machine Learning Tools and Techniques with Java Implementations. Morgan Kaufmann, San Francisco (2000)

14. Chen, S.T., Chen, G.L., Guo, W.Z., Liu, Y.H.: Feature Selection of the Intrusion Detection Data Based on Particle Swarm Optimization and Neighborhood Reduction. Journal of Computer Research and Development 47(7), 1261-1267 (2010)

15. Øhrn, A.: Rosetta Technical Reference Manual (1999), http://www.idi.ntnu.no/_aleks/rosetta

16. Banerjee, M., Mitra, S., Banka, H.: Evolutionary-Rough feature selection in gene expression data. IEEE Transactions on Systems, Man, and Cybernetics, Part C: Applications and Reviews 37, 622-632 (2007) 